\title{
Challenges in Chagas Disease Drug Discovery: a review
}

\author{
Rocio Paucar ${ }^{\dagger}$, Elsa Moreno-Viguri ${ }^{*}{ }^{\dagger}$ and Silvia Pérez-Silanes \\ Department of Organic and Pharmaceutical Chemistry, Institute for Tropical Heatlh, University of Navarra. Irunlarrea \\ $\mathrm{s} / \mathrm{n}$ 31008, Pamplona, Spain. ${ }^{\dagger}$ These authors contribute equally to this work.
}

\begin{abstract}
Chagas disease or American trypanosomiasis is a neglected tropical disease caused by the parasite Trypanosoma cruzi. Although the number of infected individuals has decreased, about 6-7 million people are infected worldwide. The chemotherapy drugs currently used are limited to benznidazole and nifurtimox. They are effective in acute phase, congenital transmission and children with chronic infection; however, recent clinical trials have shown limitations in adults with chronic infection, presenting drawbacks during the treatment. Thus, there is an urgent need for new effective, safe and affordable drugs to fight against this complex disease. There were high expectations for azole derivatives as they appeared to be the most promising drugs for the treatment of Chagas disease during the last decade; however, the disappointing results obtained so far in clinical trials evidenced the lack of correlation between preclinical and clinical development. Therefore, the feedback obtained from these studies should define the starting point for addressing a roadmap for the drug discovery process in the fight against this disease. To tackle this challenge, it is important to keep in mind the drug target profile, already defined by panels of experts, and the coordinated work involving multi-disciplinary networks focusing not only on the discovery of new drugs but also on the standardization of the protocols that would allow acceleration in the Chagas disease drug discovery process.
\end{abstract}

Keywords: Chagas disease, Trypanosoma cruzi, drug discovery, screening cascade, clinical trials, target product profile, neglected tropical disease.

\section{ACKNOWLEDGEMENTS}

RP is indebted to the University of Navarra for a grant.

\footnotetext{
*Address correspondence to this author at the Department of Organic and Pharmaceutical Chemistry, Institute for Tropical Health, University of Navarra, 31008, Pamplona, Spain; Tel/Fax: ++34 948425600; E-mail: emviguri@unav.es
} 